\title{
Regulation of MicroRNAs by Natural Products and Bioactive Compounds Obtained From Common Medicinal Plants: Novel Strategy in Cancer Therapy
}

\author{
Sevgi Gezici ${ }^{1} \&$ Nazim Sekeroglu² \\ ${ }^{1}$ Department of Biology, University of Gaziantep, Gaziantep, TURKEY, \\ ${ }^{2}$ Department of Medicinal and Aromatic Plants, University of Kilis 7 Aralik, Kilis, TURKEY
}

\begin{abstract}
MicroRNAs (miRNAs) are highly conserved non-protein coding small RNAs, known to contribute to the epigenetic regulation process in various cancer cells, including the regulation of progression, proliferation and metastasis of cancer cells, differentiation of the cells and process of apoptosis. In addition to, they are crucial for the regulation of cancer stem cells, and management the process of epithelial to mesenchymal transition of cancer cells, implicating in the cancer cells. Further to this, their roles are changeable depending on biological processes, for example miR-17, miR-20a, miR-19b-1, miR-18a, miR-19a, miR-92-1, miR-21, and miR-155 may have critical roles as oncogenic miRNAs (oncomiRs), while miR-15, miR-16, let-7, miR-34, miR-48, miR-84, miR-241 may have anticancer activities as tumor suppressor miRNAs. Therefore, the regulation of miRNAs levels by active natural compounds and/or products could be a promising strategy for cancer treatment, especially in order to inhibit cancer progression and proliferation, prevent metastasis, control the process of transition of cancer cells, and overcome chemotherapeutic resistance by increasing drug sensitivity as well. Recent studies and clinical trials which are aimed to find novel targets for the treatment of cancer, have emphasized the importance of the consumption of natural anticancer agents-rich diet. In the current review, we aimed to provide overview anticancer effects of natural compounds and/or products, isolated from medicinal plants, on miRNAs which are closely related to cancer progression and metastasis.
\end{abstract}

KEY WORDS: MicroRNAs, Cancer Therapy, Natural Products, Bioactive Molecules, Medicinal Plants, Nutri-Epigenetics.

\section{INTRODUCTION}

Cancer is a genetic disease, characterized by uncontrolled cell growth, which originates from one malignantly transformed normal cell. This cell both grows uncontrollably and multiplies quickly. Then, it spreads to other cells through body fluids such as blood and lymph nodes, which caused initiation of metastasis process. ${ }^{1,2}$ According to statistical cancer reports on the prevalence and mortality of cancer globally, cancer is reported to be the second most common disease behind cardiovascular diseases. Besides high rate of prevalence, it lead to high rate of mortality worldwide. The statistical cancer reports show that it caused almost 8.8 million deaths among a total number of 17.5 million cancer cases in 2015. Furthermore, it is estimated that the prevalence of cancer will exceed 20 million every year by 2025 , alarming fact on cancer cases. Therefore, in recent years, it has been a major and critical public health problem all over the world, it will probably continue to be more critical health problem in the following years. ${ }^{2,3}$

Even though, cancer risk and progression have been associated with many genetic and epigenetic factors such as genetic mutations, hormones, immune system, infectious
DOI: 10.5530/ijper.51.3s.71 Correspondence: Nazim Sekeroglu, Department of Medicinal and Aromatic Plants, University of Kilis 7 Aralik, Kilis, TURKEY Contact: +09 5324015512 E-Mail: nsekeroglu@gmail. com 
agents, chemicals, radiation, smoking, consumption of alcohol, obesity, cancer history. Of which epigenetic factors, not causing any alterations in DNA sequences, have been found to be more linked with initiation and/ or development of carcinogenesis. ${ }^{3,4}$ Indeed, scientific evidences have been asserted that changes and alterations in genetic and epigenetic mechanisms have significantly contributed to increase incidence and prevalence of the cancer. ${ }^{1,4}$

In the recent years, medicinal plants have offered many natural bioactive compounds that involving in prevention and treatment many cancers, as natural anticancer agents. In addition to, they have play critical roles in discovering efficient and clinically useful therapeutic anticancer agents. ${ }^{5,6}$ However, medicinal plants contain many natural products and compounds against the cancer, berberine, curcumin, genistein, daidzein, glyceollin, apigenin, quercetin, baicalein, resveratrol, luteolin, matrine, garcinol, silibinin, mangiferin, doxorubicin, and paclitaxel are common natural products and compounds found to associate with cell differentiation, proliferation and apoptosis that exhibit anticancer activities on various human cancer types. ${ }^{7-9}$

Plant derivatives natural products are capable of altering cellular signaling through epigenetic changes that include DNA methylation and histone modifications. These epigenetic changes lead to alter the expressions of miRNAs, while either increasing or decreasing of the expression levels. ${ }^{7,8}$ Because of their anticancer effects as regulating expression levels of many miRNAs, taking dietary natural products are necessary for both the inhibition of activity of oncogenic miRNAs, and inducing the activity of tumor suppressor miRNAs, which will support to inhibit development and progression of tumorogenesis. ${ }^{8-10}$

The present review is aimed to provide viewpoint concerning to elucidate the relations between the alterations in expression levels of miRNAs and process of cancer development, likewise, relation between anticancer roles of natural products and regulating effects on the expression pattern of miRNAs involved in cancer process.

\section{MicroRNAs and Their Roles in Cancer-Related Processes}

MiRNAs are small (18-25 nucleotides) and evolutionarily highly conserved non-coding RNAs that are called as the key player in gene regulation through posttranscriptional silencing or activation. ${ }^{8,11}$ Even though, miRNAs commonly localize in intergenic regions, the localization of them can differ from species to species. A single miRNA is able to target more than one hundred genes; additionally each gene contains multiple binding sides for miRNAs. ${ }^{5}$ Thus, they are involved in many multiple signaling pathways including cancer regulation processes especially, cell proliferation, and migration, metastasis, apoptosis, and cell differentiation in numerous malignancies. ${ }^{11,12}$

In terms of their functions, they are classified into two different categorizes that are tumor suppressors miRNAs which are downregulated or completely lost in cancerous conditions, and oncogenic miRNAs which are overexpressed in many cancerous cases. ${ }^{6,7}$ Among which tumor suppressing miRNAs, also called as gatekeepers, exhibit the reducing effect on tumor growth, as well as suppressing effect on the oncogenic signaling that controlled carcinogenesis mechanisms by negatively. Nevertheless, oncogenic miRNAs, also known as oncomiRs, lead to promote proto-oncogenes or block tumor suppressor genes by involving in oncogenic and tumor suppressor pathways. ${ }^{9-12}$ The expression levels of the tumor suppressor genes and oncogenes are controlled by genetic and epigenetic mechanisms that significantly associated with development of cancer pathogenesis. Methylation and acetylation are the most common posttranscriptional regulation mechanisms in human which causing alterations in the expression patterns of miRNAs. Scientific evidences showed that hyper-methylated genes become silence and inactive, while, hypo-methylated genes become active. On the other hand, recent researches have suggested that methylation status of miRNAs have strongly associated with the regulation of them, while altering the expression levels of miRNAs. ${ }^{11-13}$

Indeed, the hypo-methylation in oncogenic miRNAs causes to overexpression of the oncogene, thereby proto-oncogenes can convert to the oncogenes. Besides the activation of oncogenic miRNAs, the hyper-methylation in tumor suppressor miRNAs leads transcriptional inactivation in tumor suppressor genes. In case of any of these situations, they will lead to the loss of control in cell growth and division resulting in development of cancer proliferation and metastasis. ${ }^{13,14}$

In the recent years, scientific researches related to evaluate changes and alterations in the expression patterns of miRNAs have gained significant attention, particularly recognizing miRNA biomarkers as an indicator in various cancer cases, owing to their changeable expression levels depending on the cancerous conditions. ${ }^{7,8}$ Some of them such as miR-10b, miR-21, miR-27a, miR-155, miR-373, miR-424-5p, miR-520c have been identified as oncogenic miRNAs that found overexpressed in various cancers, whereas, the others including miR-15a, miR-16-1, miR-34, miR-126, miR-150, miR-183, miR-203, miR206, miR-335, miR-495 have been identified as tumor 
suppressor miRNAs that found down expressed in the cancers compared to normal cells. ${ }^{13-15}$

Despite the fact that many genetic and epigenetic alterations in the coding miRNA genes were determined, many of them are not tissue specific miRNA in diagnosis and prognosis of cancer, not suitable for clinical applications as well. Therefore, further researches should be focused on discovering cancer-related miRNAs which play significant roles in carcinogenic pathways.

\section{Regulation of MicroRNAs Expressions by Dietary Compounds and Natural Bioactive Products}

The expression levels of tumor suppressor miRNAs are induced, whereas, the expression levels of oncogenic
miRNAs are suppressed or silencing by bioactive compounds and natural products, found in medicinal plants as natural anticancer agents. ${ }^{5-8}$ These natural anticancer agents are obtained from various medicinal plants such as, Allium species, Brassica vegetables, Magnolia sp., Ganoderma sp., Berberis aristata, Aloe vera, Mentha longifolia, Mentha spicata L., Citrus medica, Citrus limon Burm. f., Citrus paradise Macf., Betula pendula, Mimosa pudica, Passiflora incarnate, Cannabis sativa, Azadirachta indica, Calendula officinalis, Glyccheriza glabra, Oraxylum indicum, Lithosprmum radix, Myrciaria floribunda, Thymus vulgaris, Xylopia frutescens, Neolitsea variabillima, Guatteria pogonopus, Tridax procumbens, Myrica gale, Boswellia carterii, Commiphora pyracanthoides, Amomum tsaoko, Zingiber officinale, Jasminum grandiflora,

\begin{tabular}{|c|c|c|c|c|}
\hline Bioactive compounds & $\begin{array}{l}\text { Main known medicinal } \\
\text { plants }\end{array}$ & $\begin{array}{c}\text { Target tumor } \\
\text { suppressor miRNAs }\end{array}$ & $\begin{array}{c}\text { Target oncogenic } \\
\text { miRNAs }\end{array}$ & Type of cancer \\
\hline Apigenin & $\begin{array}{c}\text { Brassica species, Allium } \\
\text { species, Matricaria } \\
\text { recutita, Capsucum } \\
\text { annuum }\end{array}$ & miR-183 & miR-103 & $\begin{array}{l}\text { Obesity and diabetes- } \\
\text { induced cancer types }\end{array}$ \\
\hline Baicalin and baicalein & Scutellaria baicalensis & miR-23a, miR-124 & $\begin{array}{l}\text { miR-181b, miR-199a-3p } \\
\text { miR-294, miR-378 }\end{array}$ & $\begin{array}{l}\text { Embryonic stem cells } \\
\text { and neuronal cells }\end{array}$ \\
\hline Berberine & $\begin{array}{l}\text { Berberis aristata, } \\
\text { Coptis japonica, } \\
\text { Coptis chinensis, } \\
\text { Phellondendron } \\
\text { amurense }\end{array}$ & $\begin{array}{c}\text { miR-21-3p, miR-34a, } \\
\text { miR-141 }\end{array}$ & $\begin{array}{l}\text { miR-21, miR-99a 125b, } \\
\text { miR-17 92, miR-106 25 }\end{array}$ & $\begin{array}{c}\text { Ovarian and breast } \\
\text { cancer, hepatocellular } \\
\text { carcinoma }\end{array}$ \\
\hline Betulinic acid & Platanus acerifolia & miR-21 & miR-27a, miR-33 & $\begin{array}{l}\text { Colon, breast and } \\
\text { hepatoma cells }\end{array}$ \\
\hline Boswellic acid and AKBA & Boswellia serrata & let-7, miR-34a, miR-200 & miR-27a & Brain and colon cancers \\
\hline Caffeine and caffeic acid & Coffea arabica & miR-124, miR-148a & $\begin{array}{l}\text { miR-9, miR-125b, miR- } \\
\text { 146a, miR-155, miR-223 }\end{array}$ & $\begin{array}{c}\text { Breast cancer and } \\
\text { hepatocarcinoma cells }\end{array}$ \\
\hline $\begin{array}{l}\text { Camptothecin (quinolone } \\
\text { alkaloid) }\end{array}$ & Camptotheca acuminata & miR-15a, miR-16 & $\mathrm{miR}-125 b$ & $\begin{array}{l}\text { Cervical carcinoma, } \\
\text { ovarian, gastric and } \\
\text { colorectal cancers }\end{array}$ \\
\hline $\begin{array}{c}\text { Catechin (tea } \\
\text { polyphenols) and EGCG }\end{array}$ & $\begin{array}{l}\text { Camelia sinensis, } \\
\text { Aspalathus linearis }\end{array}$ & $\begin{array}{c}\text { let-7b, let-7c, miR-1, } \\
\text { miR-7-1, miR-16, miR- } \\
\text { 18, miR-25, miR-34a, } \\
\text { miR-34b, miR-92, miR- } \\
\text { 99a, miR-126, miR-144- } \\
\text { 3p, miR-210, miR-330 }\end{array}$ & $\begin{array}{c}\text { miR-21, miR-30, miR-92, } \\
\text { miR-93, miR-98-5p, miR- } \\
\text { 106b, miR-374c-5p, miR- } \\
\text { 449c-5p, miR-450a-2-3p, } \\
\text { miR-453, miR-520-e, } \\
\text { miR-608, miR-629 }\end{array}$ & $\begin{array}{l}\text { Lung and prostate } \\
\text { cancers, hepatoma and } \\
\text { neuroblastoma cells }\end{array}$ \\
\hline $\begin{array}{l}\text { Curcumin (turmeric), } \\
\text { curcuminoid }\end{array}$ & Curcuma longa & $\begin{array}{c}\text { miR-7, miR-9, miR-15, } \\
\text { miR-16, miR-22, miR- } \\
\text { 29a, miR-145, } \\
\text { miR-181, miR-192-5p, } \\
\text { miR-200b/c, miR-203, } \\
\text { miR-205-5p, } \\
\text { miR-215, let-7 }\end{array}$ & $\begin{array}{l}\text { miR-17-5p, miR-20a, } \\
\text { miR-21, miR-27a, miR- } \\
\text { 186, miR-208 }\end{array}$ & $\begin{array}{c}\text { Breast, colorectal, } \\
\text { prostate and lung } \\
\text { cancers, leukemia, WT1, } \\
\text { hepatocellular carcinoma }\end{array}$ \\
\hline Doxorubicin, docetaxel & & $\begin{array}{c}\text { miR-27b, miR-34a, miR- } \\
\text { 34b, miR-101, miR-125b, } \\
\text { miR-127, miR-128, miR- } \\
\text { 193b, mir-199a, miR- } \\
\text { 200c, miR-205, miR-218, } \\
\text { miR-450b-3p, miR-451, } \\
\text { miR-497, miR-522, miR- } \\
\text { 542-3p }\end{array}$ & $\begin{array}{l}\text { miR-10b, miR-21, miR- } \\
\text { 28, miR-106a, miR-181a, } \\
\text { miR-202, miR-206, miR- } \\
\text { 221, miR-548c-3p }\end{array}$ & $\begin{array}{l}\text { Breast cancer, } \\
\text { glioblastoma and } \\
\text { lymphoma cells }\end{array}$ \\
\hline
\end{tabular}




\begin{tabular}{|c|c|c|c|c|}
\hline Bioactive compounds & $\begin{array}{l}\text { Main known medicinal } \\
\text { plants }\end{array}$ & $\begin{array}{c}\text { Target tumor } \\
\text { suppressor miRNAs }\end{array}$ & $\begin{array}{l}\text { Target oncogenic } \\
\text { miRNAs }\end{array}$ & Type of cancer \\
\hline $\begin{array}{l}\text { Garcinol and } \\
\text { Gemcitabine }\end{array}$ & Garcinia indica & $\begin{array}{l}\text { let-7, miR-453, miR-720, } \\
\text { miR-128, miR-200b/c, } \\
\text { miR-453, miR-638, miR- } \\
663, \mathrm{miR}-720, \mathrm{miR}-1280\end{array}$ & $\begin{array}{l}\text { miR-21, miR-196a, miR- } \\
\text { 495, miR-605, miR-483- } \\
\text { 3p, miR-494, miR-495, } \\
\text { miR-1914, miR-1977 }\end{array}$ & $\begin{array}{l}\text { Breast and pancreas } \\
\text { cancers }\end{array}$ \\
\hline $\begin{array}{l}\text { Genistein and soy } \\
\text { isoflavones }\end{array}$ & $\begin{array}{c}\text { Glycine max, Pueraria } \\
\text { lobata, Psoralea } \\
\text { corylifolia, Scutellaria } \\
\text { species }\end{array}$ & $\begin{array}{l}\text { let-7, miR-23b, miR-34a, } \\
\text { miR-146a, miR-200, } \\
\text { miR-574-3p, miR-1296 }\end{array}$ & $\begin{array}{l}\text { miR-21, miR-23b, miR- } \\
\text { 27a, miR-151, miR-155, } \\
\text { miR-221, miR-222, miR- } \\
\text { 223, miR-1260b }\end{array}$ & $\begin{array}{l}\text { Renal, ovarian, prostate } \\
\text { and pancreas cancers }\end{array}$ \\
\hline Glabridin & Glycyrrhiza glabra & miR-148a, miR-520a & ------ & $\begin{array}{c}\text { Hepatocellular } \\
\text { carcinoma and breast } \\
\text { cancer }\end{array}$ \\
\hline $\begin{array}{l}\text { I3C and DIM (indole } \\
\text { alkaloids) }\end{array}$ & Brassica species & \begin{tabular}{|c|} 
let-7b/c/d/e, miR-21, \\
miR-34, miR-146a, miR- \\
200b/c, miR-212/132
\end{tabular} & $\begin{array}{c}\text { miR-21, miR-31, miR-92, } \\
\text { miR-130a, miR-146b, } \\
\text { miR-221, miR-377 }\end{array}$ & Prostate and pancreas \\
\hline Luteolin & Terminalia chebula & ---- & miR-34a & Gastric cancer \\
\hline Magnolol and honokiol & Magnolia species & $\begin{array}{c}\text { miR-34a, miR-143, miR- } \\
\text { 200c }\end{array}$ & ----- & $\begin{array}{l}\text { Colon, breast and } \\
\text { bladder cancers }\end{array}$ \\
\hline Mangiferin & Mangifera indica & miR-15b, miR-182 & ----- & Lung, prostate, leukemia \\
\hline Matrine & Sophora flavescens & ----- & $\begin{array}{l}\text { let-7b-5p, miR-10a-5p, } \\
\text { miR-15b-5p, miR-18a- } \\
\text { 5p, miR-19a-3p, miR- } \\
\text { 19b-3p, miR-20b-5p, } \\
\text { miR-21, mir-21-5p, miR- } \\
\text { 23a-3p, miR-26b-5p, }\end{array}$ & $\begin{array}{l}\text { Gastric and breast } \\
\text { cancers }\end{array}$ \\
\hline Paclitaxel (taxol) & Taxus brevifolia & $\begin{array}{c}\text { let-7b, miR-9, miR-10b, } \\
\text { miR-16, miR-17, miR-17- } \\
\text { 5p, miR-34a, miR-122, } \\
\text { miR-125a, miR-130a, } \\
\text { miR-134, miR-141, miR- } \\
\text { 155, miR-200c, miR-218, } \\
\text { miR-367, miR-494, miR- } \\
\text { 497, miR-647, miR-873, } \\
\text { miR-877, miR-1204 }\end{array}$ & $\begin{array}{c}\text { miR-19a, miR-21, miR- } \\
\text { 22, miR-30a-5p, miR- } \\
\text { 129-5p, miR-130b, miR- } \\
\text { 149, miR-153, } \\
\text { miR-133a/b, miR-197, } \\
\text { miR-205, miR-320a, } \\
\text { miR-361-3p, miR-375, } \\
\text { miR-433, miR-490, } \\
\text { miR-520h, miR-622, } \\
\text { miR-663, miR-1307 }\end{array}$ & $\begin{array}{c}\text { Ovarian, breast and lung } \\
\text { cancers }\end{array}$ \\
\hline Palmitine & Coptis japonica & $\begin{array}{c}\text { miR-34a, miR-141, miR- } \\
\text { 200c }\end{array}$ & ----- & $\begin{array}{l}\text { Prostate and breast } \\
\text { cancers }\end{array}$ \\
\hline Quercetin & $\begin{array}{c}\text { Allium species, Malus } \\
\text { domestica, Phaseolus } \\
\text { vulgaris, Vitis vinifera, } \\
\text { Capparis sicula, Citrus } \\
\text { species }\end{array}$ & $\begin{array}{c}\text { let-7, miR-16, miR-26, } \\
\text { miR-34a, miR-142-3p, } \\
\text { miR-146a }\end{array}$ & miR-27a, miR-125b-3p & $\begin{array}{l}\text { Colon, lung and } \\
\text { pancreas cancers }\end{array}$ \\
\hline Resveratrol & $\begin{array}{c}\text { Arachis hypogaea, } \\
\text { Muscadine berries, Vitis } \\
\text { vinifera }\end{array}$ & $\begin{array}{l}\mathrm{miR}-34 a / c, \text { miR-122- } \\
5 p, \mathrm{miR}-141, \mathrm{miR}-194 \\
\text { miR-200c, miR-299-5p, } \\
\text { miR-338-3p, miR-582- } \\
\text { 3p, miR-622, miR-663, } \\
\text { miR-758, miR-774 }\end{array}$ & $\begin{array}{l}\text { miR-17, miR-21, miR-25, } \\
\text { miR-27a, miR-92a-2, } \\
\text { miR-103-1, miR-103-2, } \\
\text { miR-183, miR-200-3p, } \\
\text { miR-520h, miR-542-3p, } \\
\text { miR-17-92 }\end{array}$ & $\begin{array}{l}\text { Colorectal, lung, } \\
\text { pancreas, prostate and } \\
\text { gastric cancers }\end{array}$ \\
\hline Rosmarinic acid & Rosmarinus officinalis & miR-124, miR-148a & $\begin{array}{l}\text { miR-9, miR-125b, miR- } \\
\text { 146a, miR-155, miR-223 }\end{array}$ & $\begin{array}{l}\text { Brain, breast and lung } \\
\text { cancers }\end{array}$ \\
\hline Silibinin & Silybum marianum & $\mathrm{miR}-200 \mathrm{c}$ & miR-21 & Lung cancers \\
\hline Ursolic acid & $\begin{array}{l}\text { Radix actinidiae, } \\
\text { Oldenlandia diffusa }\end{array}$ & ----- & miR-21 & Glioma cells, \\
\hline $\begin{array}{c}\text { Vinca alkaloids } \\
\text { (vinblastine, vincristine) }\end{array}$ & Catharanthus roseus & $\begin{array}{c}\text { miR-25, miR-34a, miR- } \\
\text { 93, miR-93, miR-199a, } \\
\text { miR-224, miR-424, miR- } \\
\text { 494, miR-497 }\end{array}$ & $\begin{array}{c}\text { miR-27a, miR-27b, miR- } \\
\text { 148a, miR-324-3p, miR- } \\
\text { 328, miR-451 }\end{array}$ & $\begin{array}{c}\text { B-cell lymphoma, breast, } \\
\text { prostate and laryngeal } \\
\text { cancers }\end{array}$ \\
\hline
\end{tabular}


Nectandra megapotamica, Matricaria chamomilla, Rosa damascena, Zanthoxylum rboifolium Lam., Lavandula angustifolia, Lavandula stoechas ssp. stoechas, Talauma ovata, Symphyopappus itatiayensis, Psidium cattleianum, Juniperus phoenicea, Malus domestica, Comptonia peregrina L., Artemisia indica, Litsea cubeba, Casearia sylvestris, Photinia serrulata and Plectranthus amboinicus. ${ }^{13-18}$ The miRNAs regulated by a selection of important anticancer natural products and compounds that obtained from main known medicinal plants are presented in Table 1.

As summarized in Table 1, recent studies and clinical trials which are aimed to find novel targets for the treatment of multiple types of cancers, have emphasized the importance of the consumption of natural agents-rich diet, including curcumin, berberine, alkaloids, mitomycin C, resveratrol, camptothecin, topotecan, trabectedin, matrine, honokiol, isoflovones, indole-3-carbinol, sulforaphane, water soluble vitamins, diallyl disulfide, 3,3'-diindolylmethane, epigallocatechin-3-gallate and hesperidin, etc., additionally syntheses of natural products by the liposome or nanoparticle formulations assays. . $^{5,16-18}$

The bioactive compounds and natural products have significant influences on the epigenetic changes in the cancer cells. For instance, they regulate the expression levels of miRNAs by effecting the several proteins that are involved in the stages of tumorogenesis both cancer initiation and development. ${ }^{17,18}$ Apart from, they effect cell signaling and apoptotic pathways such as PI3K-AktmTOR, AR, Akt and ERK signaling, Wnt signaling, p53, Erb and MAPK signaling, Src/Ras/ERK and TGF- $\beta$ signaling. From this point of view, intake of these natural agents by dietary regimes appears worthwhile for effective cancer therapy as a nutri-epigenetic approach, since approximately $30-35 \%$ cancer, a substantial proportion, can be prevented by dietary choices. ${ }^{1,10,11}$ In addition, dietary interventions give opportunity to cure more efficiently with fewer side effects and toxicity. ${ }^{78}$

\section{CONCLUSIONS AND FUTURE PERSPECTIVES \\ MicroRNAs as Novel Biomarkers in Cancer}

Consequently, regulation of microRNAs by natural products and/or bioactive compounds obtained from common medicinal plants would likely provide a novel therapeutic agent for cancer therapy in the following years particularly, when provided to be certain that there are no side effects of dietary intake.

\section{ACKNOWLEDGEMENT}

The authors gratefully acknowledge Professor Raman Dang and Dr. Sonal Dubey for their kind scientific and technical supports.

\section{CONFLICT OF INTERESTS}

The authors have declared that no conflict of interest exists.

\section{ABBREVIATIONS}

AKBA: 3-acetyl-11-keto-b-boswellic acid; Akt: a serine/ threonine kinase also known as protein kinase $\mathrm{B}[\mathrm{PKB}]$; AR: androgen receptor; DIM: 3,30-diindolylmethane; EGCG: [-] epigallocatechin-3-gallate; ERK: extracellular signal-regulated kinase; I3C: indole-3-carbinol; mTOR: mammalian target of rapamycin; p53: tumor protein 53; PI3K: phosphatidylinositol 3-kinase; TGF- $\beta$ : transforming growth factor beta.

\section{REFERENCES}

1. Siegel RL, Miller KD, Jemal A. Cancer statistics, 2015. CA: a cancer journal for clinicians. 2015;65(1):5-29.

2. Manju K, Jat R, Anju G. A review on medicinal plants used as a source of anticancer agents. International Journal of Drug Research and Technology. 2017;2(2):6.

3. Fitzmaurice C, Allen C, Barber RM, Barregard L, Bhutta ZA, Brenner H, et al. Global, regional, and national cancer incidence, mortality, years of life lost, years lived with disability, and disability-adjusted life-years for 32 cancer groups, 1990 to 2015: a systematic analysis for the global burden of disease study. JAMA oncology. 2017;3(4):524-48.

4. Danaei G, Hoorn VS, Lopez AD, Murray CJ, Ezzati M, group CRAc. Causes of cancer in the world: comparative risk assessment of nine behavioural and environmental risk factors. The Lancet. 2005;366(9499):1784-93.

5. Biersack B. Current state of phenolic and terpenoidal dietary factors and natural products as non-coding RNA/microRNA modulators for improved cancer therapy and prevention. Non-coding RNA Research. 2016;1(1):12-34.

6. Hong M, Wang N, Tan HY, Tsao S-W, Feng Y. MicroRNAs and Chinese medicinal herbs: New possibilities in cancer therapy. Cancers. 2015;7(3):1643-57.

7. Devi KP, Rajavel T, Daglia M, Nabavi SF, Bishayee A, Nabavi SM, editors. Targeting miRNAs by polyphenols: Novel therapeutic strategy for cancer. Seminars in Cancer Biology; 2017: Elsevier.

8. Biersack B. Non-coding RNA/microRNA-modulatory dietary factors and natural products for improved cancer therapy and prevention: Alkaloids, organosulfur compounds, aliphatic carboxylic acids and water-soluble vitamins. Non-coding RNA Research. 2016;1(1):51-63.

9. Sala-Cirtog $M$, Marian $C$, Anghel A. New insights of medicinal plant therapeutic activity-the miRNA transfer. Biomedicine \& Pharmacotherapy. 2015;74:228-32.

10. Palmer JD, Soule BP, Simone BA, Zaorsky NG, Jin L, Simone NL. MicroRNA expression altered by diet: can food be medicinal? Ageing research reviews. 2014;17:16-24.

11. Thakur VS, Deb G, Babcook MA, Gupta S. Plant phytochemicals as epigenetic modulators: role in cancer chemoprevention. The AAPS journal. 2014;16(1):151-63.

12. Sethi S, Li Y, H Sarkar F. Regulating miRNA by natural agents as a new strategy for cancer treatment. Current drug targets. 2013;14(10):1167-74.

13. Lujambio A, Calin GA, Villanueva A, Ropero S, Sánchez-Céspedes M, Blanco $\mathrm{D}$, et al. A microRNA DNA methylation signature for human cancer metastasis. Proceedings of the National Academy of Sciences. 2008;105(36):13556-61. 
14. Swierczynski S, Klieser E, Illig R, Alinger-Scharinger B, Kiesslich T, Neureiter D. Histone deacetylation meets miRNA: epigenetics and post-transcriptional regulation in cancer and chronic diseases. Expert opinion on biological therapy. 2015;15(5):651-64.

15. Li Y, Kong D, Wang Z, Sarkar FH. Regulation of microRNAs by natural agents: an emerging field in chemoprevention and chemotherapy research. Pharmaceutical research. 2010;27(6):1027-41.

16. Xie W, Weng A, Melzig MF. MicroRNAs as new bioactive components in medicinal plants. Planta medica. 2016;82(13):1153-62.
17. Feitelson MA, Arzumanyan A, Kulathinal RJ, Blain SW, Holcombe RF, Mahajna J, et al., editors. Sustained proliferation in cancer: Mechanisms and novel therapeutic targets. Seminars in cancer biology; 2015: Elsevier.

18. Jiang $Q$, Han $Y$, Gao H, Tian R, Li P, Wang C. Ursolic acid induced antiproliferation effects in rat primary vascular smooth muscle cells is associated with inhibition of microRNA-21 and subsequent PTEN/PI3K. European journal of pharmacology. 2016;781:69-75.

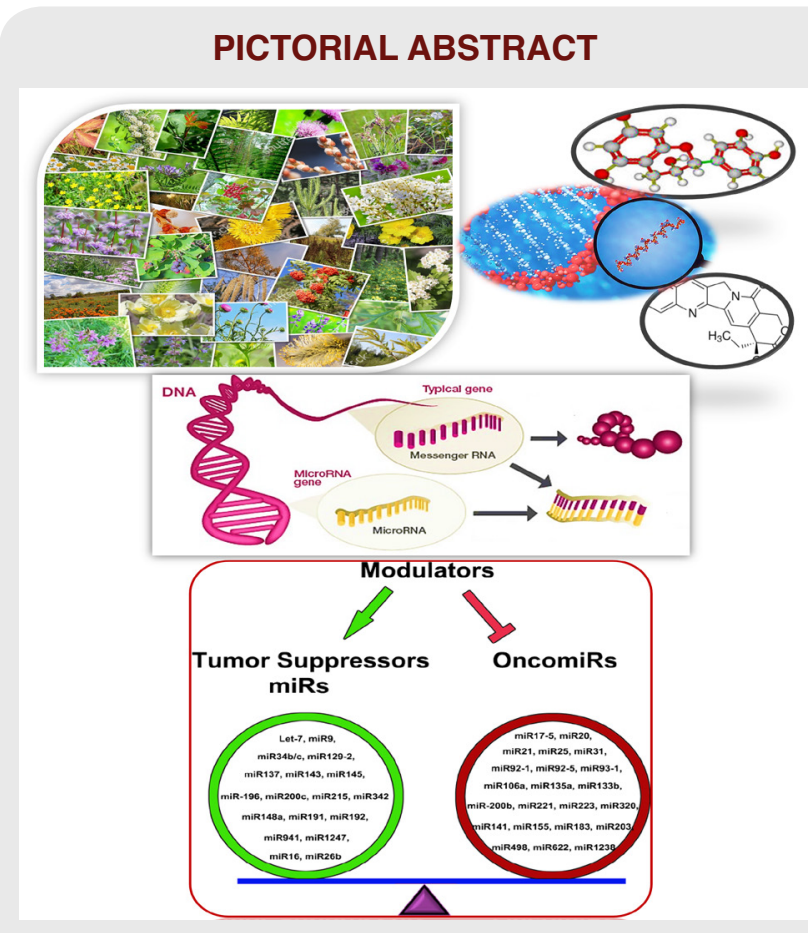

\section{SUMMARY}

- MicroRNAs (miRNAs) contribute to the epigenetic regulation process in progression, proliferation and metastasis of cancer cells.

- They exhibit changeable expression levels depending on the biological process, so they play critical roles in cancerous conditions as oncogenic miRNAs (oncomiRs) or tumor suppressor miRNAs.

- Their expression levels can be suppressed or induced by active natural compounds and/or products, isolated from MAPs.

- In the current review, we aimed to provide overview anticancer effects of natural compounds and/or products, isolated from MAPs, to emphasize the importance of the consumption of natural agents-rich diet for both cancer prevention and treatment as well.

- Overall, MAPs would likely provide a novel therapeutic strategy for cancer therapy in the following years particularly, when provided to be certain that there are no side effects of dietary intake

\section{ABOUT AUTHORS}

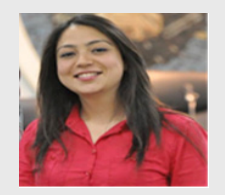

Dr. Sevgi Gezici: After completing her Ms in Molecular Biology and Genetic at Department of Biology, Gaziantep University, Turkey, she earned her Ph.D degree in the Molecular Biology and Genetics form the same University. She has received scholarships for her MSc and Ph.D from TUBITAK, which is the best research center in Turkey. She is currently doctor at Gaziantep University, Department of Molecular Biology.

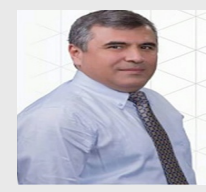

Prof. Dr. Nazim Sekeroglu: He earned his BsC in Agricultural Engineering, Prof. Dr. Sekeroglu earned his PhD degree on Medicinal and Aromatic Plants Cukurova University, Turkey. He is interested in medicinal and aromatic plants since his undergraduate education. He is currently a full-time professor at Kilis 7 Aralik University, Department of Medicinal and Aromatic Plants. He published over 100 peer-reviewed articles and still active on his research area. Additionally, he has organized many international scientific meetings, including congress, symposiums and workshops as chairperson. Furthermore, he is the founder of the Association of medicinal and Aromatic Plants of Mediterranean (AMAPMED), also he is general coordinator of Global Federation of Medicinal and Aromatic Plants (GOFMAP), a worldwide non-profit organization gathering associations related 'Medicinal and Aromatic Plants'. Moreover, he is a member of scientific commissions about Medicinal and Aromatic Plants at Food, Agriculture, Forestry and Health Ministries in Turkey. He has visited more than 50 different countries for scientific purposes.

Cite this article: Gezici S \& Sekeroglu N. Regulation of Micrornas by Natural Products and Bioactive Compounds Obtained From Common Medicinal Plants: Novel Strategy in Cancer Therapy. Indian J of Pharmaceutical Education and Research. 2017;51(3)Suppl:S483-88. 